\title{
Primary malignant melanoma of the esophagus
}

\author{
Chunxiang Ling ${ }^{1}$ (D) Jizhen Feng ${ }^{1}$ (D) Jiamei Li ${ }^{2}$ (D), Qingwei Liu' (D) \\ ${ }^{1}$ Department of Radiology, Shandong Provincial Hospital Affiliated to Shandong University, Jinan, China \\ 2Department of Pathology, Shandong Provincial Hospital Affiliated to Shandong University, Jinan, China
}

Cite this article as: Ling C, Feng J, Li J, Liu Q. Primary malignant melanoma of the esophagus. Turk J Gastroenterol 2018; 29: 711-3.

\section{Dear Editor,}

Primary malignant melanoma of the esophagus (PMME) is a rare disease that accounts for $0.1 \%-0.2 \%$ of the total primary malignant tumors of the esophagus (1).

A 62-year-old Chinese male presented with a 1-month history of dysphagia for solids accompanied by odynophagia. There was no past history of cutaneous, ocular, or other site melanomas. An esophagogastroduodenoscopy (EGD) performed in our outpatient department revealed a polypoid tumor in the lower esophagus, at a site $33 \mathrm{~cm}$ from the incisors, accompanied by hyperemia and erosion (Figure 1). An endoscopic biopsy of the lesion was performed. The samples were sent for a pathological examination, which revealed melanoma (Figure 2). A double-contrast esophagogram showed an irregular filling defect localized in the lower esophagus, expanding the distal esophagus without causing obstruction. A contrast-enhanced (CE)

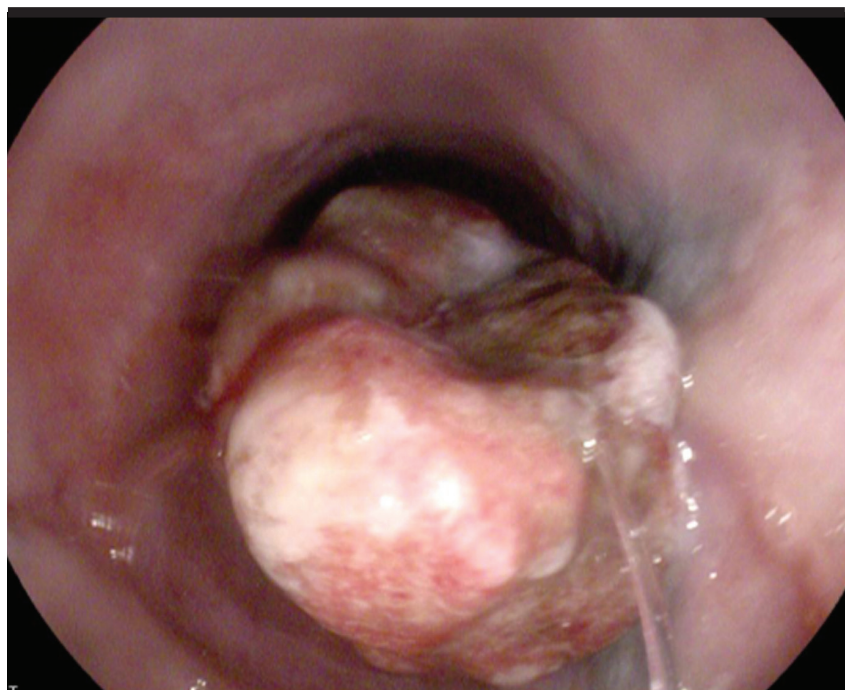

Figure 1. EGD revealing a polypoid tumor with hyperemia and erosion in the lower esophagus computed tomography (CT) scan of the thorax and the abdomen revealed the presence of eccentric thickening of the lower esophagus wall, with a moderate enhancement (Figure 3). There were no apparent metastases. Therefore, an esophagectomy with extensive lymph node dissection was performed, and the specimen was sent for pathological examination. The diagnosis was PMME. The patient had a consistent fever 1 day after surgery due to lung infection and hydrothorax, and he requested to be discharged home 25 days after the surgery without palliative chemotherapy, radiotherapy, and a routine CT follow-up because of the poor prognosis of the disease and financial constraints. The patient being in a poor postoperative condition still only eats liquid food and is followed up via telephone. A written informed consent was obtained from the patient for his participation in this study.

Primary malignant melanoma of the esophagus is a rare disease that accounts for $0.1 \%-0.2 \%$ of the total pri-

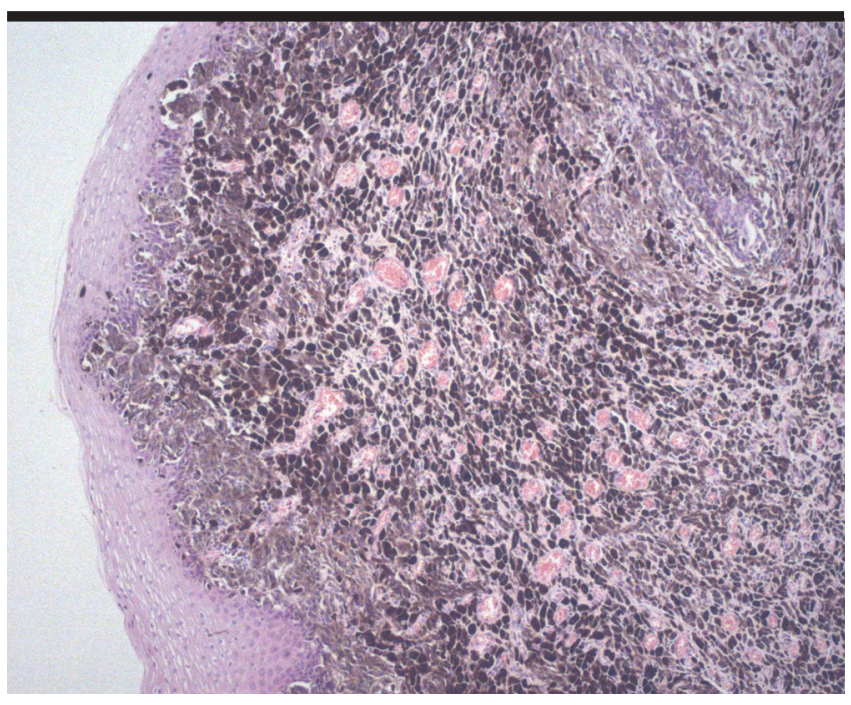

Figure 2. Histopathological features of esophageal malignant melanoma (H\&E staining, $\times 100)$

ORCID IDs of the authors: C.L. 0000-0002-7267-3744; J.F. 0000-0002-6748-5191; J.L. 0000-0003-2752-5678; Q.L. 0000-0002-6343-9067.

Corresponding Author: Qingwei Liu; doctorlqw@163.com

Received: January 19, 2018 Accepted: March 21, 2018 Available online date: August 2, 2018

(C) Copyright 2018 by The Turkish Society of Gastroenterology · Available online at www.turkjgastroenterol.org

DOI: 10.5152/tjg.2018.18065 


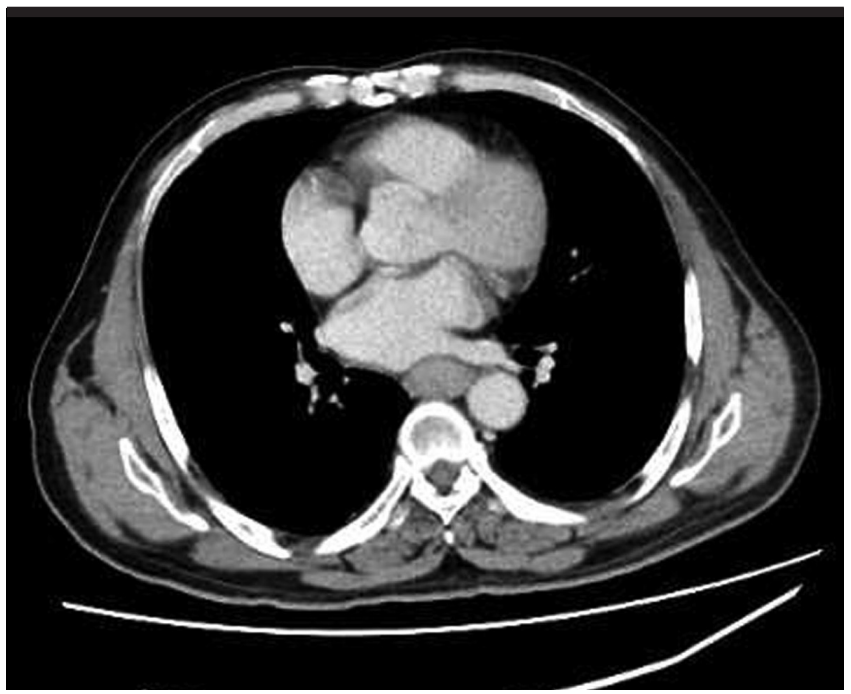

Figure 3. A CE-CT scan demonstrating an eccentric thickening in the lower third of the esophagus wall, with a moderate enhancement

mary malignant tumors of the esophagus. It most often occurs in men, and most patients are in their sixth and seventh decades (1). Dysphagia and epigastralgia are the chief complaints of patients with PMME (2). Nonspecific clinical symptoms are easily ignored, which delays the diagnosis. In approximately $90 \%$ of the PMME cases, the most frequent location is in the middle or the distal third of the esophagus probably because of a greater concentration of melanocytes in these regions $(1,3)$.

A double-contrast esophagogram in PMME usually reveals a smooth-surface tumor that is lobulated, polyploid, protruding, or sectionalized and has a tendency of growing in the radial or horizontal directions. The tumor region is often distended, which makes it different from other esophageal malignant tumors (2).

A CE-CT examination demonstrates the esophageal lesion with regard to its size, shape, degree of local spread, lymph node invasion, and remote metastatic disease. Magnetic resonance can facilitate preoperative diagnosis because it detects high-signal-intensity masses in the esophagus on T1-weighted imaging because of the paramagnetic scavenging by melanin. A positron emission tomography and computed tomography (PET/CT) examination is also useful in the PMME treatment program particularly to evaluate the effect of postoperative therapy and search for occult metastasis (4-6). In our case, the patient declined PET/CT examination because of financial constraints.
Positive findings of S100 protein and HMB45 by immunohistochemical staining are sometimes necessary to diagnose PMME in certain cases, particularly when an ordinary histologic examination is insufficient $(2,7)$.

The differential diagnosis of PMME includes squamous cell carcinoma, spindle cell carcinoma, leiomyosarcoma, lymphoma, rhabdomyosarcomas, epidermoid and small cell carcinoma, Kaposi's sarcoma, and adenocarcinoma arising from a Barrett's esophagus. It is hard to differentiate these tumors based on radiological signatures, but there are some manifestations that can point to some of them.

Primary malignant melanoma of the esophagus prognosis is fatal, with a median survival rate of 34.5 months after surgery, regardless of the treatment (8). The only therapeutic option that impacts survival is surgical treatment. The most important prognostic factor and predominant death cause is the presence of metastases $(8,9)$. Therefore, because tumor cells longitudinally disseminate, a total or near-total esophagectomy is essential (10).

Primary malignant melanoma of the esophagus is a rare tumor with fatal prognosis. It is hard to distinguish it from non-epithelial malignant tumors because of the limited information supplied by radiological images. Therefore, the diagnosis of PMME should combine clinical symptoms, auxiliary examination, pathological examination, and immunohistochemistry.

Informed Consent: Informed consent was obtained from the patient who participated in this study.

Peer-review: Externally peer-reviewed.

Author Contributions: Concept - C.L., Q.L.; Design - C.L., J.F.; Supervision - J.F., Q.L.; Resources J.L., Q.L.; Materials - C.L., J.L.; Data Collection and/or Processing - C.L., J.F.; Analysis and/or Interpretation C.L., J.F.; Literature Search - C.L., Q.L.; Writing Manuscript - C.L.; Critical Review - J.F., J.L., Q.L.

Conflict of Interest: The authors have no conflict of interest to declare.

Financial Disclosure: This study was supported by grants from Natural Science Foundation of China (\#81502568).

\section{REFERENCES}

1. Sabanathan S, Eng J, Pradhan G N. Primary malignant melanoma of the esophagus. Am J Gastroenterol 1989; 84: 1475-81.

2. Iwanuma Y, Tomita N, Amano T, et al. Current status of primary malignant melanoma of the esophagus: clinical features, pathol- 
ogy, management and prognosis. J Gastroenterol 2012; 47: 21-8. [CrossRef]

3. Yoo C C, Levine M S, McLarney J K, Lowry M A. Primary malignant melanoma of the esophagus: radiographic findings in seven patients. Radiology 1998; 209: 455-9. [CrossRef]

4. Stranks, Mathai, Rowe-Jones. Primary malignant melanoma of the oesophagus: case report and review of surgical pathology. Gut 1991; 32: 828-30. [CrossRef]

5. Lin $P$, Lee $R$, Chern $M$, Chiang J, Chang C. Primary malignant melanoma of the esophagus. J Chin Med Assoc 2006; 69: 334-7. [CrossRef]

6. Zheng J, Mo H, Ma S, Wang Z. Clinicopathological findings of primary esophageal malignant melanoma: report of six cases and review of literature. Int J Clin Exp Pathol 2014; 7: 7230-5.
7. Joob AW, Haines GK 3rd, Kies MS, Shields TW. Primary malignant melanoma of the esophagus. Ann Thorac Surg 1995; 60: 217-22. [CrossRef]

8. Harada K, Mine S, Yamada K, et al. Long-term outcome of esophagectomy for primary malignant melanoma of the esophagus: a single-institute retrospective analysis. Dis Esophagus 2016; 29: 314-9. [CrossRef]

9. Li B, Lei W, Shao K, et al. Characteristics and prognosis of primary Malignant melanoma of the esophagus. Melanoma Res 2007; 17: 239-42. [CrossRef]

10. Volpin E, Sauvanet A, Couvelard A, Belghiti J. Primary malignant melanoma of the esophagus: a case report and review of the literature. Dis Esophagus 2002; 15: 244-9. [CrossRef] 